\title{
Generalized similarity method in unsteady two-dimensional MHD boundary layer on the body which temperature varies with time
}

\section{Dragisa Nikodijevic ${ }^{1}$, Zoran Boricic ${ }^{1}$, Dragica Milenkovic ${ }^{1}$, Zivojin Stamenkovic ${ }^{{ }^{*}}$}

\author{
${ }^{1}$ University of Nis, Faculty of Mechanical Engineering, Aleksandra Medvedeva 14, 18000 Nis, Serbia
}

"Corresponding Author: e-mail:zikas@masfak.ni.ac.rs

\begin{abstract}
In this paper, the multiparametric method known as generalized similarity method is used to solve the problem of unsteady temperature two-dimensional MHD laminar boundary layer of incompressible fluid. It is assumed that outer magnetic field induction is function only from longitudinal coordinate. Magnetic field acts perpendicular to the body on which boundary layer forms. Body temperature varies with time. Further, electric field is neglected and value of magnetic Reynolds number is significantly less then one i.e. problem is considered in induction-less approximation. According to temperature differences under $50^{\circ} \mathrm{C}$ physical properties of fluid are constant. Introduced assumptions simplify considered problem in sake of mathematical solving, but adopted physical model is interesting from practical point of view, because its relation with large number of technically significant MHD flows. Obtained partial differential equations can be solved with modern numerical methods for every particular problem. In this paper, quite different approach is used. In the first place new variables are introduced and then similarity parameters which enable transformation of equations into universal form. Obtained universal equations and corresponding boundary conditions do not contain explicit characteristics of particular problems. Based on obtained universal equations, approximated universal differential equations of described MHD boundary layer flow problem are derived. Aproximated universal equations do not depend on the particular problems.
\end{abstract}

Keywords: boundary layer, MHD, generalized similarity method, electroconductive fluid

\section{Introduction}

Idea of boundary layer control appear when Prandtl form the theory, and this idea came from Prandtl himself (Schlichting, 1982). Boundary-layer control usually means either attempts to change the overall flowfield to reduce pressure drag and/or to increase lift or attempts to control the position of boundary layer separation point. For a long time following methods was used for boundary layer control: increasing the boundary layer velocity, boundary layer suction, second gas injection, profile laminarization, body cooling.

Interest in effect of outer magnetic filed on heat-physical processes appear sixty years ago (Blum et al., 1966). Developing of this research was stimulated by two problems: protection of spacecrafts from aerodynamic overheating and destruction during the passage through dense atmosphere layers; building the operational ability of high temperature MHD generators constructive elements for direct transformation of heat energy in to electricity. First problem show that magnetohydrodynamical influence on ionized gases is convenient control method for mass, heat and hydrodynamic processes. Solutions of mentioned problems were followed with rapid increase of analytical papers and experimental procedures about heat transfer in MHD boundary layer (Blum et al., 1980, Askovic et al., 1977).

MHD devices for liquid metals engage metallurgist attention. It was shown that effect of magnetic filed could be very helpful in modernization of technological processes. Developing of nuclear power systems is almost unconceivable without usage of MHD devices. Controlling of crystallization processes in metallurgy and influence of magnetic field on discrete chemical systems bring MHD and heat physics in relation with problems that was research subject of physical chemistry, kinetics, biophysics... At the 
end, analogies, which appear with knowledge of magnetic field influence on mechanics and biological suspensions (especially blood), brought to possibility to transfer heat physics research results into magneto-biological and medical processes (Tzirtzilakis, 2005).

\section{Mathematical model}

In this paper, for the sake of richness of mentioned research, unsteady temperature two-dimensional laminar MHD boundary layer of incompressible electro-conductive fluid is studied. It is assumed that outer magnetic filed induction is function of longitudinal coordinate $\mathbf{B}=B(x) \vec{j}$. Magnetic field acts perpendicular to the body surface on which boundary layer forms. Further, it is presumed there is no outer electric filed and magnetic Reynolds number is significantly lower then one i.e. considered problem is in induction-less approximation (induced magnetic field due to the fluid motion is neglected). Velocity of flow is considered much lower then speed of light and usual assumption in thermal boundary layer calculation that temperature difference is small (Schlichting, 1982) is used; accordingly characteristic properties of fluid (density, viscosity...) are constant. Introduced assumptions (Soundalgekar et al., 1990) simplify considered problem, however obtained physical model is interesting from practical point of view, because its relation with large number of MHD flows significant for technical practice. Described MHD problem is mathematically presented with continuity equation:

$$
\frac{\partial u}{\partial x}+\frac{\partial v}{\partial y}=0
$$

moment equation:

$$
\frac{\partial u}{\partial t}+u \frac{\partial u}{\partial x}+v \frac{\partial u}{\partial y}=\frac{\partial U}{\partial t}+U \frac{\partial U}{\partial x}+v \frac{\partial^{2} u}{\partial y^{2}}-\frac{\sigma B^{2}}{\rho}(u-U)
$$

energy equation:

$$
\frac{\partial T}{\partial t}+u \frac{\partial T}{\partial x}+v \frac{\partial T}{\partial y}=\frac{\lambda}{\rho c_{p}} \frac{\partial^{2} T}{\partial y^{2}}+\frac{\mu}{\rho c_{p}}\left(\frac{\partial u}{\partial y}\right)^{2}+\frac{\sigma B^{2}}{\rho c_{p}}(u-U)^{2}
$$

and corresponding boundary and initial conditions:

$$
\begin{aligned}
& u=0, v=0, T=T_{w}(t) \text { for } y=0 ; \\
& u \rightarrow U(x, t), T \rightarrow T_{\infty} \text { for } y \rightarrow \infty ; \\
& u=u_{0}(x, y), T=T_{0}(x, y) \text { for } t=t_{0} ; \\
& u=u_{1}(t, y), T=T_{1}(t, y) \text { for } x=x_{0} .
\end{aligned}
$$

For further consideration stream function, $\Psi(x, y, t)$ is introduced with following relations:

$$
\frac{\partial \Psi}{\partial x}=-v, \frac{\partial \Psi}{\partial y}=u
$$

which satisfies equation (1) identically and transform moment equation (2) into equation:

$$
\frac{\partial^{2} \Psi}{\partial t \partial y}+\frac{\partial \Psi}{\partial y} \frac{\partial^{2} \Psi}{\partial x \partial y}-\frac{\partial \Psi}{\partial x} \frac{\partial^{2} \Psi}{\partial y^{2}}=\frac{\partial U}{\partial t}+U \frac{\partial U}{\partial x}+v \frac{\partial^{3} \Psi}{\partial y^{3}}-\frac{\sigma B^{2}}{\rho}\left(\frac{\partial \Psi}{\partial y}-U\right)
$$

and energy equation into equation: 


$$
\frac{\partial T}{\partial t}+\frac{\partial \Psi}{\partial y} \frac{\partial T}{\partial x}-\frac{\partial \Psi}{\partial x} \frac{\partial T}{\partial y}=\frac{\lambda}{\rho c_{p}} \frac{\partial^{2} T}{\partial y^{2}}+\frac{\mu}{\rho c_{p}}\left(\frac{\partial^{2} \Psi}{\partial y^{2}}\right)^{2}+\frac{\sigma B^{2}}{\rho c_{p}}\left(\frac{\partial \Psi}{\partial y}-U\right)^{2}
$$

Boundary and initial conditions are transformed into equations:

$$
\begin{aligned}
& \Psi=0, \frac{\partial \Psi}{\partial y}=0 ; T=T_{w}(t) \text { for } y=0 ; \\
& \frac{\partial \Psi}{\partial y} \rightarrow U(x, t) ; T \rightarrow T_{\infty} \text { for } y \rightarrow \infty ; \\
& \frac{\partial \Psi}{\partial y}=u_{0}(x, y), T=T_{0}(x, y) \text { for } t=t_{0} ; \\
& \frac{\partial \Psi}{\partial y}=u_{1}(t, y), T=T_{1}(t, y) \text { for } x=x_{0} .
\end{aligned}
$$

In the case of flow around a body boundary conditions for third order equation (9) are conditions (11) and (12). First two conditions ( $\Psi=0, \partial \Psi / \partial y=0)$ means: line $y=0$ coincides with zero streamline ( $\Psi=0)$; longitudinal velocity is equal to zero on the body $(u=\partial \Psi / \partial y=0)$. Third boundary condition $\partial \Psi / \partial y \rightarrow U(x, t)$ means asymptotic approaching of longitudinal velocity to the velocity on outer edge of boundary layer. Condition $v=-\partial \Psi / \partial y=0$ for $y=0$ is consequence of equality $\Psi=0$ on body surface. Equation (9) does not depend from equation (10and it can be solved independently. Solution of equation (9) is used for solving of equation (10).

\section{Universal equations}

Obtained partial differential equations can be solved for every particular case using modern numerical methods and computer. In this paper, quite different approach is used based on ideas in papers (Skadov, 1963; Loicijanski, 1965; Saljnikov, 1978; Busmarin et al., 1974), which is extended in papers (Boricic et al., 2005; Obrovic et al., 2005; Nikodijevic et al., 2009). Essence of this approach is in introducing adequate transformations and sets of parameters in starting equations, which transform the equations system and corresponding boundary conditions into form unique for all particular problems and this form is considered as universal. Solution of universal equations can be used to derive general conclusions about developing of temperature MHD boundary layer and also for special cases calculation. Integration of obtained universal equations is performed once for all. In order to solve particular problems it is necessary to determine impulse equation using obtained universal solutions. In order to realize described procedure following new variables are introduced:

$$
\begin{aligned}
& x=x, t=t, \eta=\frac{D y}{h(x, t)} \\
& \Phi(x, t, \eta)=\frac{D \Psi(x, y, t)}{U(x, t) h(x, t)}, \quad \Theta(x, t, \eta)=\frac{T_{w}-T}{T_{w}-T_{\infty}}
\end{aligned}
$$

where $D$ is normalizing constant, and $h(x, t)$ is characteristic linear scale of transversal coordinate in boundary layer. According to introduced variables, equation (9) is transformed in new form:

$$
D^{2} \frac{\partial^{3} \Phi}{\partial \eta^{3}}+f_{1,0}\left(\Phi \frac{\partial^{2} \Phi}{\partial \eta^{2}}-\left(\frac{\partial \Phi}{\partial \eta}\right)^{2}+1\right)+\left(f_{0,1}+g_{1,0}\right)\left(1-\frac{\partial \Phi}{\partial \eta}\right)+\frac{1}{2}(F \Phi+\eta g) \frac{\partial^{2} \Phi}{\partial \eta^{2}}=z \frac{\partial^{2} \Phi}{\partial t \partial \eta}+U z X(\eta ; x)
$$

where for the sake of shorter expression, the notations are introduced: 


$$
\begin{aligned}
& z=\frac{h^{2}}{v} ; g=\frac{\partial z}{\partial t} ; N=\frac{\sigma B^{2}}{\rho} ; g_{1,0}=N z \\
& F=U \frac{\partial z}{\partial x} ; f_{1,0}=z \frac{\partial U}{\partial x} ; f_{0,1}=\frac{z}{U} \frac{\partial U}{\partial t} ; \\
& X\left(x_{1} ; x_{2}\right)=\frac{\partial \Phi}{\partial x_{1}} \frac{\partial^{2} \Phi}{\partial \eta \partial x_{2}}-\frac{\partial \Phi}{\partial x_{2}} \frac{\partial^{2} \Phi}{\partial x_{1} \partial \eta}
\end{aligned}
$$

and equation (10) now have the form:

$$
\begin{aligned}
& \frac{D^{2}}{P_{r}} \frac{\partial^{2} \Theta}{\partial \eta^{2}}-D^{2} E_{c}\left(\frac{\partial^{2} \Phi}{\partial \eta^{2}}\right)^{2}-E_{c} g_{1,0}\left(1-\frac{\partial \Phi}{\partial \eta}\right)^{2}+(1-\Theta) l_{1}+ \\
& +\frac{1}{2} \eta g \frac{\partial \Theta}{\partial \eta}+\frac{1}{2}\left(F+2 f_{1,0}\right) \Phi \frac{\partial \Theta}{\partial \eta}=z \frac{\partial \Theta}{\partial t}-U z Y(x ; \eta)
\end{aligned}
$$

where the following notations have been used for the sake of shorter statement:

$$
\begin{aligned}
& \operatorname{Pr}=\frac{v \rho c_{p}}{\lambda}-\text { Prandtl number; } \\
& E_{c}=\frac{U^{2}}{c_{p}\left(T_{w}-T_{\infty}\right)} \text {-Eckert number; } \\
& l_{1}=\frac{Z}{T_{w}-T_{\infty}} \frac{d T_{w}}{d t} ; \\
& Y\left(x_{1} ; x_{2}\right)=\frac{\partial \Phi}{\partial x_{1}} \frac{\partial \Theta}{\partial x_{2}}-\frac{\partial \Phi}{\partial x_{2}} \frac{\partial \Theta}{\partial x_{1}} .
\end{aligned}
$$

Now we introduce sets of parameters: dynamical $f_{k, n}$, magnetic $g_{k, n}$, temperature $l_{k}$, and constant parameter $g$, as new independent variables:

$$
\begin{aligned}
& f_{k, n}=U^{k-1} \frac{\partial^{k+n} U}{\partial x^{k} \partial t^{n}} z^{k+n} \quad(k, n=0,1,2, \ldots ; k \vee n \neq 0) ; \\
& g_{k, n}=U^{k-1} \frac{\partial^{k-1+n} N}{\partial x^{k-1} \partial t^{n}} z^{k+n} \quad(k, n=0,1,2, \ldots, ; k \neq 0) ; \\
& l_{k}=\frac{1}{q} \frac{\partial^{k} q}{\partial x^{k}} z^{k} \quad(k=1,2, \ldots) ; \\
& g=\frac{\partial z}{\partial t}=\text { const. }
\end{aligned}
$$

where:

$$
q=T_{w}-T_{\infty} .
$$


Introduced sets of parameters reflect the nature of velocity change on outer edge of boundary layer, alteration characteristic of variable $N$ and temperature change on body surface, and a part from that, in the integral form (by means of $z$ and $\partial z / \partial t$ ) prehistory of flow in boundary layer. These parameters enable transformations of differential equations (16) and (18) into universal form in sense that neither equations nor boundary conditions explicitly depends from values that characterized particular problems. Procedure for obtaining "universal" equations has following steps. First, we find the derivates in Eqs. (16) and (18) using the operators given in Eqs. (25)-(26), and then transform these equations with introduced independent variables $\eta ; f_{k, n} ; g_{k, n} ; l_{k, n}$. For derivate along longitudinal coordinate $x$ and for time derivate next operators are used:

$$
\begin{gathered}
\frac{\partial}{\partial x}=\sum_{\substack{k, n=0 \\
k \vee n \neq 0}}^{\infty} \frac{\partial f_{k, n}}{\partial x} \frac{\partial}{\partial f_{k, n}}+\left\{\begin{array}{l}
0, \text { for } \Phi \\
\sum_{k=1}^{\infty} \frac{\partial l_{k}}{\partial x} \frac{\partial}{\partial l_{k}}, \text { for } \Theta+\sum_{\substack{k=1 \\
n=0}}^{\infty} \frac{\partial g_{k, n}}{\partial x} \frac{\partial}{\partial g_{k, n}} ;
\end{array}\right. \\
\frac{\partial}{\partial t}=\sum_{\substack{k, n=0 \\
k \vee n \neq 0}}^{\infty} \frac{\partial f_{k, n}}{\partial t} \frac{\partial}{\partial f_{k, n}}+\left\{\begin{array}{l}
0, \text { for } \Phi \\
\sum_{k=1}^{\infty} \frac{\partial l_{k}}{\partial t} \frac{\partial}{\partial l_{k}}, \text { for } \Theta+\sum_{\substack{k=1 \\
n=0}}^{\infty} \frac{\partial g_{k, n}}{\partial t} \frac{\partial}{\partial g_{k, n}} .
\end{array}\right.
\end{gathered}
$$

Parameter derivates along coordinate $x$ and time $t$ are obtained by differentiation of Eqs. (20)-(22):

$$
\begin{aligned}
& \frac{\partial f_{k, n}}{\partial x}=\frac{1}{U z}\left\{(k-1) f_{1,0} f_{k, n}+(k+n) F f_{k, n}+f_{k+1, n}\right\}=\frac{1}{U z} Q_{k, n} \\
& \frac{\partial f_{k, n}}{\partial t}=\frac{1}{z}\left\{(k-1) f_{0,1} f_{k, n}+(k+n) g f_{k, n}+f_{k, n+1}\right\}=\frac{1}{z} E_{k, n} \\
& \frac{\partial g_{k, n}}{\partial x}=\frac{1}{U z}\left\{(k-1) f_{1,0} g_{k, n}+(k+n) F g_{k, n}+g_{k+1, n}\right\}=\frac{1}{U z} K_{k, n} \\
& \frac{\partial g_{k, n}}{\partial t}=\frac{1}{z}\left\{(k-1) f_{0,1} g_{k, n}+(k+n) g g_{k, n}+g_{k, n+1}\right\}=\frac{1}{z} L_{k, n} ; \\
& \frac{\partial l_{k}}{\partial x}=\frac{1}{U z}\left\{k F l_{k}\right\}=\frac{1}{U z} M_{k} ; \\
& \frac{\partial l_{k}}{\partial t}=\frac{1}{z}\left\{\left(k g-l_{1}\right) l_{k}+l_{k+1}\right\}=\frac{1}{z} N_{k}
\end{aligned}
$$

where $Q_{k, n} ; E_{k, n} ; K_{k, n} ; L_{k, n} ; M_{k} ; N_{k}$ are terms in curly brackets in obtained equations. It is important to notice $Q_{k, n} ; K_{k, n} ; M_{k}$ beside the parameters depend on value $U \partial z / \partial x=F$. Using parameters (20)-(23) instead coordinate $x$ and time $t$, operators (25) and (26), and terms (27)-(32) Eqs. (16) and (18) are transformed into equations:

$$
\begin{aligned}
\mathfrak{J}_{1}=\sum_{\substack{k, n=0 \\
k \vee n \neq 0}}^{\infty}\left[Q_{k, n} X\left(\eta ; f_{k, n}\right)+E_{k, n} \frac{\partial^{2} \Phi}{\partial \eta \partial f_{k, n}}\right]+\sum_{\substack{k=1 \\
n=0}}^{\infty}\left[K_{k, n} X\left(\eta ; g_{k, n}\right)+L_{k, n} \frac{\partial^{2} \Phi}{\partial \eta \partial g_{k, n}}\right] ; \\
\mathfrak{I}_{2}=\sum_{\substack{k, n=0 \\
k \vee n \neq 0}}^{\infty}\left[Q_{k, n} Y\left(\eta ; f_{k, n}\right)+E_{k, n} \frac{\partial \Theta}{\partial f_{k, n}}\right]+\sum_{k=1}^{\infty}\left[M_{k} Y\left(\eta ; l_{k}\right)+N_{k} \frac{\partial \Theta}{\partial l_{k}}\right]+ \\
+\sum_{\substack{k=1 \\
n=0}}^{\infty}\left[K_{k, n} Y\left(\eta ; g_{k, n}\right)+L_{k, n} \frac{\partial \Theta}{\partial g_{k, n}}\right]
\end{aligned}
$$


where the following markings have been used for shorter statement: $\mathfrak{\Im}_{1}$-left side of equation (16), $\mathfrak{I}_{2}$ - left side of equation (18). From the Eqs. (33) and (34) can be seen, the characteristics of external flow dominate by the means of function $F$ which appears in terms for $Q_{k, n} ; K_{k, n} ; M_{k}$. In order the equations (33) and (34) to be independent of the outer flow characteristics i.e. to be universal, it is necessary to show the existence of the equality $F=F\left[\left(f_{k, n}\right),\left(g_{k, n}\right),\left(l_{k}\right)\right]$.

So as to show the existence of such equality, we start from the momentum equation:

$$
\frac{\partial}{\partial t}\left(U \delta^{*}\right)+\frac{\partial}{\partial x}\left(U^{2} \delta^{* *}\right)+U\left(\frac{\partial U}{\partial x}+N\right) \delta^{*}-\frac{\tau_{w}}{\rho}=0
$$

in which:

$$
\begin{aligned}
& \delta^{*}(x, t)=\int_{0}^{\infty}\left(1-\frac{u}{U}\right) d y \text {-displacement thickness; } \\
& \delta^{* *}(x, t)=\int_{0}^{\infty} \frac{u}{U}\left(1-\frac{u}{U}\right) d y \text {-momentum thickness; } \\
& \tau_{w}(x, t)=\mu\left(\frac{\partial u}{\partial y}\right)_{y=0} \text {-friction stress on the body. }
\end{aligned}
$$

Introducing dimensionless characteristic functions:

$$
\begin{aligned}
& H^{*}(x, t)=\frac{\delta^{*}}{h} ; \\
& H^{* *}(x, t)=\frac{\delta^{* *}}{h} ; \\
& \xi(x, t)=\frac{\tau_{w} h}{\mu U}
\end{aligned}
$$

which, according to Eqs. (15) and (36)-(38), can be expressed in the following form:

$$
\begin{aligned}
& H^{*}(x, t)=\frac{1}{D} \int_{0}^{\infty}\left(1-\frac{\partial \Phi}{\partial \eta}\right) d \eta \\
& H^{* *}(x, t)=\frac{1}{D} \int_{0}^{\infty} \frac{\partial \Phi}{\partial \eta}\left(1-\frac{\partial \Phi}{\partial \eta}\right) d \eta \\
& \xi(x, t)=\left.D \frac{\partial^{2} \Phi}{\partial \eta^{2}}\right|_{\eta=0} .
\end{aligned}
$$

After transition to new independent variables values $H^{*}, H^{* *}, \xi$ become functions only from parameters $f_{k, n}, g_{k, n}, l_{k, n}, g$. Now, using parameters as new independent variables and derivative operators from impulse Eq. (35) after simple transformations next equation is obtained: 


$$
F=\frac{P}{Q}
$$

where, $P$ and $Q$ are:

$$
\begin{gathered}
P=\xi-f_{1,0}\left(2 H^{* *}+H^{*}\right)-\left(f_{0,1}+g_{1,0}+\frac{1}{2} g\right) H^{*}-\sum_{\substack{k, n=0 \\
k \vee n \neq 0}}^{\infty}\left\{E_{k, n} \frac{\partial H^{*}}{\partial f_{k, n}}+\left[(k-1) f_{1,0} f_{k, n}+f_{k+1, n}\right] \frac{\partial H^{* *}}{\partial f_{k, n}}\right\} \\
\quad-\sum_{\substack{k=1 \\
n=0}}^{\infty}\left\{L_{k, n} \frac{\partial H^{*}}{\partial g_{k, n}}+\left[(k-1) f_{1,0} g_{k, n}+g_{k+1, n}\right] \frac{\partial H^{* *}}{\partial g_{k, n}}\right\} ; \\
Q=\frac{1}{2} H^{* *}+\sum_{\substack{k, n=0 \\
k \vee n \neq 0}}^{\infty}(k+n) f_{k, n} \frac{\partial H^{* *}}{\partial f_{k, n}}+\sum_{\substack{k=1 \\
n=0}}^{\infty}(k+n) g_{k, n} \frac{\partial H^{* *}}{\partial g_{k, n}} .
\end{gathered}
$$

Last two equations define function $F$ in terms of values, which depends only from introduced parameters. Eqs. (33) and (34) with respect to (41)-(43) are now universal equations of described problem. Since the system of Eqs. (33) and (34) do not contain explicitly: the free stream velocity distribution, outer magnetic field, body temperature and the temperature of the free stream which defines each particular case of considered flow, these equations are considered as universal (independent from outer flow characteristic). Boundary conditions, also universal, are given with terms:

$$
\begin{aligned}
& \Phi=0, \frac{\partial \Phi}{\partial \eta}=0, \Theta=0 \text { for } \eta=0 \\
& \Phi \rightarrow 1, \Theta \rightarrow 1 \text { for } \eta \rightarrow \infty \\
& \Phi=\Phi_{0}(\eta), \Theta=\Theta_{0}(\eta) \text { for }\left\{\begin{array}{ll}
f_{k, n}=0,(k, n=0,1,2, \ldots k \vee n \neq 0) \\
g_{k, n}=0 & (k, n=0,1,2, \ldots, k \neq 0) \\
l_{k}=0 & (k=1,2, \ldots) \\
g=0 &
\end{array}\right\} ;
\end{aligned}
$$

where $\Phi_{0}(\eta)$ is Blasius solution for stationary boundary layer on the plate, $\Theta_{0}(\eta)$ is solution of following equation:

$$
\frac{D^{2}}{\operatorname{Pr}} \frac{d^{2} \Theta_{0}}{d \eta^{2}}-D^{2} E c\left(\frac{d^{2} \Phi_{0}}{d \eta^{2}}\right)^{2}+\frac{\xi_{0}}{H^{* *}} \Phi_{0} \frac{d \Theta_{0}}{d \eta}=0
$$

Universal Eqs. (33) and (34) with boundary conditions (44)-(46) are exact for wide class of problems in which $z=A t+C(x)$, where $A$ is arbitrary constant and $C(x)$ some function of longitudinal coordinate. For other problems this equations are approximated "universal" equations.

Eqs. (33) and (34) are integrated in m-parametric approximation once for good and all. Obtained characteristic function can be used to yield general conclusions about development of described boundary layer and to solve any particular problem. Before integration for scale of transversal coordinate in boundary layer $h(x, t)$ some characteristic value is chosen. In this case $h=\delta^{* *}$ and Eq. (41) now have form: 


$$
F=2\left[\xi-f_{1,0}(2+H)-\left(f_{0,1}+g_{1,0}+\frac{1}{2} g\right) H-\sum_{\substack{k, n=0 \\ k \vee n \neq 0}}^{\infty} E_{k, n} \frac{\partial H}{\partial f_{k, n}}-\sum_{\substack{k=1 \\ n=0}}^{\infty} L_{k, n} \frac{\partial H}{\partial g_{k, n}}\right]
$$

Taking parameters $f_{k, n}=0, g_{k, n}=0, g=0$ Eq. (33) is simplified into form:

$$
\frac{d^{3} \Phi_{0}}{d \eta^{3}}+\frac{\xi_{0}}{D^{2}} \Phi_{0} \frac{d^{2} \Phi_{0}}{d \eta^{2}}=0
$$

which describe boundary layer flow on flat plate. Previous equation must coincide with well known Blasisus equation, which repute for exact equation and according to previous statement it is necessary to chose $D^{2}=\xi_{0}$. Using exact Blasisus solution (Schlichting, 1982) $\xi_{0}=0.2209$ for further investigations normalizing constant $D=0.47$ must be chosen (Loicijanski, 1965, White, 1994). For selected value $h$ Eq. (47) for determining variable $\Theta_{0}$ became:

$$
\frac{1}{\operatorname{Pr}} \frac{d^{2} \Theta_{0}}{d \eta^{2}}+\Phi_{0} \frac{d \Theta_{0}}{d \eta}-E c\left(\frac{d^{2} \Phi_{0}}{d \eta^{2}}\right)^{2}=0
$$

Eqs. (33) and (34) contain on the right sides infinite sums. In order to solve these equations it is necessary to limit number of independent variables. This leads to the implementation of "snipping" method, which implies equalization of variables with zero starting form some index. In this way approximated equations are derived. For higher number of detained parameters-variables, approximated equations are more precise. In this paper approximations of Eqs. (33) and (34) are given in which influence of parameters $f_{1,0}, f_{0,1}, g_{1,0}, l_{1}$, and $g$ are detained and influence of parameters $f_{0,1}, l_{1}$ derivatives are disregarded. Approximation of Eqs. (33) and (34) are given with following terms:

$$
\begin{aligned}
& \mathfrak{I}_{1}=F f_{1,0} X\left(\eta ; f_{1,0}\right)+g f_{1,0} \frac{\partial^{2} \Phi}{\partial \eta \partial f_{1,0}}+F g_{1,0} X\left(\eta ; g_{1,0}\right)+g g_{1,0} \frac{\partial^{2} \Phi}{\partial \eta \partial g_{1,0}} \\
& \mathfrak{I}_{2}=F f_{1,0} Y\left(\eta ; f_{1,0}\right)+g f_{1,0} \frac{\partial \Theta}{\partial f_{1,0}}+F g_{1,0} Y\left(\eta ; g_{1,0}\right)+g g_{1,0} \frac{\partial \Theta}{\partial g_{1,0}}
\end{aligned}
$$

and function $F$ is obtained from Eq. (48) in same approximation:

$$
F=2\left[\xi-f_{1,0}(2+H)-\left(f_{0,1}+g_{1,0}+\frac{1}{2} g\right) H-g g_{1,0} \frac{\partial H}{\partial g_{1,0}}-g f_{1,0} \frac{\partial H}{\partial f_{1,0}}\right] .
$$

Boundary conditions which coincide to system of Eqs. (51) and (52) are conditions (44), (45) and condition:

$$
\Phi=\Phi_{0}(\eta), \Theta=\Theta_{0}(\eta) \text { for } f_{1,0}=0, f_{0,1}=0, g_{1,0}=0, l_{1}=0, g=0
$$

which is obtained from condition (46), using same simplifications like as equations. Universal equations need to be solved with corresponding boundary conditions (44), (45) and (54) using three-diagonal method, known in Russian literature as the "progonka" method. Solving of these equations is subject of further research. Obtained universal solutions can be used for general conclusions about boundary layer development and also for calculations for every particular problem.

\section{Summary}

In this paper unsteady thermal two-dimensional MHD boundary layer is considered. This problem can be analyzed for every particular case i.e. for given outer flow characteristics. Here is used quite different approach in order to use benefits of multiparametric method and universal equations of observed problem are derived. The independence of the obtained system of equations and boundary conditions of particular velocity and temperature distribution at the edge of boundary layer; of outer 
magnetic field and of body temperature enable the integration of these equations, once for good and all. In the procedure of integration, the velocity and temperature dimensionless profiles $\Phi, \Theta$ in cross-stream sections of boundary layer, friction coefficient $\xi$, and also the characteristic functions $H^{*}, H^{* *}, F$ in the dependence of dynamical $f_{k, n}$, magnetic $g_{k, n}$ and temperature $l_{k}$ parameters can be determined. That obtained so-called universal solutions should, in appropriate manner, be kept and be used not only for drawing the general conclusions about the unsteady thermal MHD boundary layer development, but also for the calculation of the particular problems. Surely, the universal equations can be used only in the some corresponding approximation, which assumes the finite number of terms on the right-hand sides of these equations. Approximation is in relation with taking into account definite number of parameters and one example of approximated equations are given in paper. Solving of these equations and results analysis is subject of future research.

\section{Nomenclature}

$\begin{array}{lll}B & {[T]} & \text { magnetic field induction } \\ C_{p} & {[\mathrm{~J} / \mathrm{kgK}]} & \text { specific heat capacity } \\ E c & {[-]} & \text { Eckert number } \\ F & {[-]} & \text { characteristic function } \\ f_{k, n} & {[-]} & \text { dynamical parameters } \\ g_{k, n} & {[-]} & \text { magnetic parameters } \\ H & {[-]} & \text { characteristic function } \\ H^{*} & {[-]} & \text { characteristic function } \\ H^{* *} & {[-]} & \text { characteristic function } \\ l_{k, n} & {[-]} & \text { temperature parameters } \\ N & {\left[\mathrm{~s}^{-1}\right]} & \text { characteristic function } \\ P r & {[-]} & \text { Prandtl number } \\ q & {[\mathrm{~K}]} & \text { temperature difference between body surface and outer flow } \\ t & {[\mathrm{~s}]} & \text { time } \\ T & {[\mathrm{~K}]} & \text { thermodynamic temperature } \\ u, v & {[\mathrm{~m} / \mathrm{s}]} & \text { longitudinal and transversal velocity in boundary layer respectively } \\ U & {[\mathrm{~m} / \mathrm{s}]} & \text { velocity on outer edge of boundary layer } \\ x, y & {[\mathrm{~m}]} & \text { longitudinal and transversal coordinate respectively } \\ Z & {[\mathrm{~s}]} & \text { characteristic function } \\ \delta^{*} & {[\mathrm{~m}]} & \text { extrusion thickness } \\ \delta^{* *} & {[\mathrm{~m}} & \text { thickness of impulse loss } \\ \Phi & {[-]} & \text { dimensionless stream function } \\ \eta & {[-]} & \text { dimensionless transversal coordinate } \\ \lambda & {\left[\mathrm{W} / \mathrm{mK}^{\prime}\right.} & \text { thermal conductivity } \\ \mu & {\left[\mathrm{P}_{a} \mathrm{~s}\right]} & \text { viscosity } \\ v & {\left[\mathrm{~m}^{2} / \mathrm{s}\right]} & \text { kinematic viscosity } \\ \Theta & {[-]} & \text { dimensionless temperature difference } \\ \rho & {\left[\mathrm{kg} / \mathrm{m}^{3}\right]} & \text { fluid density } \\ \sigma & {\left[A^{2} \mathrm{~s}^{3} / \mathrm{kgm}^{3}\right] \text { conductivity }} \\ \tau & {\left[\mathrm{P}_{a}\right]} & \text { shear stress } \\ \Psi & {\left[\mathrm{m}^{2} / \mathrm{s}\right]} & \text { stream function } \\ \xi & {[-]} & \text { characteristic function } \\ & & \end{array}$

\section{Subscripts and Superscripts}

$0 \quad$ initial time moment

1 known boundary layer cross-section

$\infty \quad$ outer boundary of boundary layer

w body surface 


\section{References}

Ašković T., Ašković R., 1977. Sur un probleme de la couche limite magnetohydrodynamique laminare non stationnaro avec une conductivite electrique variable, ZAMM Vol. 57, pp. 170-173.

Blum E.J., Mihailov J.A. and Ozols R.J., 1980. Heat and mass transfer in magnetic field (in Russian), SSR Latvian science academy, Physics institute, Zinatie, Riga.

Blum E.J., Mihailov J. A., 1966. Heat transfer in electroconductive fluid in presence of transversal magnetic field (in Russian), Fifth edition of magnetohydrodynamics-Turbulent MHD flow, Riga, pp. 2-18.

Boricic, Z., Nikodijevic, D., Milenkovic, D., Stamenkovic, Z., 2005, A form of MHD universal equations of unsteady incompressible fluid flow with variable electroconductivity on heated moving plate, Theoretical and Applied Mechanics, Vol.32, No.1, pp. 65-77.

Busmarin O.N., Saraev J.V., 1974. Parametric method in theory of unsteady boundary layer (in Russian), Inz.-fiz. zurnal, Vol. XXVII, pp. 110-118.

Loicijanski L.G., 1965. Universal equations and parametric approximations in theory of laminar boundary layer (in Russian), AN SSSR Applied Mathematics and Mechanics, Vol. 29, pp. 70-87.

Obrovic, B., Nikodijevic, D., Savic S., 2005, Boundary layer of the dissociated gas flow over a porous wall under the conditions of equilibrium dissociation, Theoretical and Applied Mechanics., Vol.32, No.2, pp. 165-190.

Nikodijevic, D., Boricic, Z., Blagojevic, B. and Stamenkovic, Z. 2009. Universal solutions of unsteady two-dimensional MHD boundary layer on the body with temperature gradient along surface, WSEAS Transactions on Fluid Mechanics, Issue 3, Vol. 4, pp. 97-106.

Saljnikov, V. N., 1978, A contribution to universal solutions of the boundary layer theory, Theoretical and Applied Mechanics Vol. 4, pp. 139 - 163.

Schlichting H., 1982. Grenzschicht-Theorie, Braun-Verlag, Karlsruhe.

Skadov V., 1963. Boundary layer flow of compressible fluid with pressure gradient (in Russian), Science Academy OTN Mechanics and Mechanism, Vol. 2, pp. 28-32.

Soundalgekar V.M.,Ramana Murty T.V, and Takhar N.S., 1990, Heat transfer in MHD unsteady stagnation point flow with variable wall temperature, Indian Journal of Pure and Applied Mathematics, Vol. 21-4, pp. 384-389.

Tzirtzilakis, E. E., 2005, A mathematical model for blood flow in magnetic field, Physics of Fluids, Vol. 17 Issue 7, p. 15. White, F., 1994, Viscous fluid flow, McGraw-Hill, New York.

\section{Biographical notes}

Dr. Dragisa Nikodijevic is Professor at the Department of Fluid Mechanics, Faculty of Mechanical Engineering, Nis, Serbia. He has engaged in teaching and research activities since the last 30 years. He completed two faculties Mechanical Engineering and Mathematics. His field of specialization is magnetohydordynamic (MHD) boundary layer flows. Dr. D. Nikodijevic has published more than 100 papers in various national, international conferences and journals. He was Vice Dean of the Mechanical Engineering Faculty more then 10 years.

Dr. Zoran Boricic is Professor \& Head, Department of Fluid Mechanics, Faculty of Mechanical Engineering, Nis, Serbia. He has engaged in teaching and research activities since the last 40 years. During his stay at the Chair for Aerodynamics LPI-Russia, under the tutorial of the world famous scientist L. G. Locjansky, he completed his Ph.D relating to the study of magnetohydrodynamic flow by applying modern numerical integration methods. Dr. Z. Boricic has published more than 100 papers in various national, international conferences and journals. He was many times elected for Dean of the Mechanical Engineering Faculty.

Dr. Dragica Milenkovic is working as a Professor in Fluid Mechanics Department, Faculty of Mechanical Engineering, Nis, Serbia. She has done research in the field of unsteady flow in turbo machinery. She has published severe number of papers on various research areas such as renewable energy, turbomachinery design and analysis of unsteady flow regimes, in national and international journals. Presently, she is a Vice Dean of Mechanical Engineering Faculty.

Zivojin Stamenkovic is $\mathrm{Ph}$. D. student. He completed the studies and presently he is focusing on doctor thesis in the field of magnetohydordynamic flow of micropolar fluids. He is engaged in two projects for Ministry of technology and development in research of MHD devices development and design of centrifugal pumps with improved suction characteristics. He has more then twenty international publication in conference proceedings, national and international journals.

Received October 2009

Accepted November 2009

Final acceptance in revised form December 2009 